\title{
Modelling and Analysis on Emergency Evacuation from Metro Stations
}

\author{
Shaokuan Chen, Yue Di, Shuang Liu, and Baoshan Wang \\ The MOE Key Laboratory for Transportation Complex Systems Theory and Technology, Beijing Jiaotong University, \\ Beijing 100044, China \\ Correspondence should be addressed to Shaokuan Chen; shkchen@bjtu.edu.cn
}

Received 3 January 2017; Revised 20 April 2017; Accepted 3 May 2017; Published 24 May 2017

Academic Editor: Jian G. Zhou

Copyright (C) 2017 Shaokuan Chen et al. This is an open access article distributed under the Creative Commons Attribution License, which permits unrestricted use, distribution, and reproduction in any medium, provided the original work is properly cited.

\begin{abstract}
Efficient calculation on passenger evacuation performances under emergencies is crucial to the planning, design, and operation of metro systems as it is closely related to the safety of human lives. Four calculation methodologies including the China CDM, NFPA 130 , control volume, and $\mathrm{M} / \mathrm{G} / \mathrm{c} / \mathrm{c}$ models and a multiagent-based simulation approach are employed to calculate the evacuation time under a given number of evacuees in different application backgrounds by investigating impact factors and strategies of evacuation as well as moving speed of evacuees. The M/G/c/c model and multiagent-based simulation approach are also used to identify the bottleneck of evacuation in metro stations. A case study for the Xizhimen metro station in Beijing is carried out to validate the effectiveness of these methodologies to planners, designers, and operators in practice. The results show that the former two methodologies are concise quantitative methods while the latter ones are more capable of assessing the whole process and details of evacuation.
\end{abstract}

\section{Introduction}

Many large cities in China have to meet the problems of severe traffic congestion and pollution. They urgently need the construction and operations of metro systems. These large cities have operated dozens of large metro networks which include 133 metro lines amounting to 4152.8 kilometres and 2680 metro stations by the end of 2016. It is worthwhile to note that the metro systems both in Beijing and in Shanghai have more than 350 stations, which are required to provide guaranteed transportation service for over nine million passengers per day. Many crowded stations are experiencing very high density of passengers which probably leads to a large risk of accidents during operations.

Accidents like fire, explosion, or toxic gas release in buildings like stadiums, shopping malls, or transportation terminals always threaten human lives. The accidents occurring in metro stations have also led to severe life and social economic loss since the first metro line was put into operation in 1860s. One of the important reasons leading to huge losses from accidents is the inefficient performances and unadvisable strategies of passenger evacuation after the accidents occurred. For example, an improper evacuation arrangement that a train stopped by the platform where the other train had caught fire made the train suffer a more severe damage and led to heavy casualties accounting for $92 \%$ of all deaths in the Daegu metro fire [1]. The investigation on evacuation performances under reasonable strategies, especially the evacuation time and evacuee number, thus becomes much essential to evaluate the safety of passengers during the design and operation of metro systems.

Approaches including analytical methods and simulation for pedestrian evacuation in public buildings have been paid extensive attention [2-4]. The methodologies employed in pedestrian evacuation mainly include optimization, simulation, and risk assessment models [5]. They mainly include cellular automata models [6], lattice gas models [7], social force models [8], fluid dynamic models [9], innovative models [10], and experimental approaches with animals [11]. Although some of the above methodologies were adopted for the evacuation problems in metro systems [12], the emphasis of evacuation research concentrated on modelling movement 
characteristics [13], evaluating facility capacity [14, 15] and investigating strategy scenarios $[16,17]$ through field data collection and scenario simulation.

Analytical methods are appropriate for the smallscale evacuation problems through depicting evacuation behaviour [18] while elaborate microscopic simulators necessitate a complex modelling process and a large amount of collected data and calibration parameters [5]. Moreover, the aims and interests of research on evacuation are much distinct according to task requirements and application background. For instance, it is important for the designers and managers of metro stations to know that all evacuees are able or unable to be evacuated under a given configuration of facilities through a simple and efficient analytical method while technical personnel of researchers are required to identify that all evacuees are safe or unsafe during the evacuation by microscopic simulation.

The analytical methodologies and simulation are necessary and competent to evaluate emergency evacuation by collecting and calibrating parameters in metro stations where there are several exits, many facilities, complex layouts, and heavy transportation load. To distinguish their advantages/ disadvantages, this research investigates four calculation methods and one practical simulation approach considering different influence factors and requirements for evacuation to evaluate evacuation time and evacuee number and then compares the applicability of these methodologies based on field data through case studies.

\section{Emergency Evacuation in Metro Stations}

The calculation and simulation for emergency evacuation mainly pay attention to impact factors [15] and evacuation strategies [17] which, respectively, define the microscopic and macroscopic mechanisms of evacuation problems.

2.1. Impact Factors of Evacuation. The impact factors of evacuation in metro stations mainly include individual physiological and psychological characteristics of passengers as well as capacity of facilities.

2.1.1. Individual Characteristics. The evacuation behaviour is greatly affected by individual physiological and psychological characteristics. It is also affected by the density of crowd and emotional state. A double exponential relationship between free movement speed and emotional state was obtained through extreme value theory [19]. It revealed that the level of emotional response increases with the urgency of evacuation while the attention and control of individuals can rapidly decrease. As a result, passengers subconsciously try to move faster than normal. Moreover, competition and congestion appear because of a lack of coordination between strong desire for survival and evacuation bottleneck [14]. Helbing et al. recommended an efficient movement speed around $1.5 \mathrm{~m} / \mathrm{s}$ through their dynamical model considering panic [2] which was also observed and validated in China [20].

2.1.2. Capacity of Facilities. The facilities related to evacuation in metro stations mainly include staircases and escalators on platforms and concourses, connecting corridors, automatic fare gate, staircases, and escalators at the exits. Some design guidelines recommend the evacuation capacities for these facilities, such as China CDM (China Code for Design of Metro) [21] and NPFA 130 (National Fire Protection Association 130) [22] in USA. Unfortunately, these facilities are not always utilized efficiently, especially under emergency circumstances. The staircase and escalators connecting platforms usually become crowding or congestion bottlenecks over peak hours according to field observation and existing experiences.

2.2. Evacuation Strategies. Evacuation strategies should be first determined when an evacuation scheme is proposed. The numbers of evacuees, evacuation routes, and terminus are indispensable to evacuation strategies.

2.2.1. Evacuee Number. The number of passengers being able to evacuate within a certain period is one of the most crucial evacuation indices. It is considered and calculated in terms of the most adverse condition. The maximum evacuee number in China include passengers from a full loaded train or referring to the maximum cross section of passenger flow, waiting and walking passengers on platforms.

2.2.2. Evacuation Route. If a fatal accident happens in a metro station, all evacuees have to quickly evacuate from the accident source and other involved areas. An overall emergency evacuation direction and route is given as shown in Figure 1.

China CDM defines the operation state of the facilities when all evacuees leave along an evacuation route in emergency circumstances [21].

(i) Train and screen doors are required to open fully for passengers and Term 9.7.12 also requires that emergency escape doors be opened to all evacuees during platform accidents.

(ii) Term 18.3.4 requires that all fare gates be always kept into passing mode for passengers when emergencies happen or power is off.

(iii) Terms 28.2.12 requires that planners or designers consider the breakdown of one escalator which cannot be utilized by passengers and also requires that the capacity of staircases and escalators be calculated by $90 \%$ of their normal capacity.

2.2.3. Evacuation Terminus. Evacuation terminuses are usually defined by evacuation safe spots which actually are different places according to emergency positions. For a train fire and platform fire, concourse or corridors can be defined as the safe spots of evacuation in some design guidelines as ventilation systems can prevent smoke from evacuees. For a concourse fire, evacuation terminuses may not be located in metro stations and depend on how the ventilation systems effectively operate in underground or overhead stations. The joint areas between a concourse and entrance/exit corridors can generally be regarded as a temporary safe spot. However, the entrances or exits of metro stations are always evacuation 


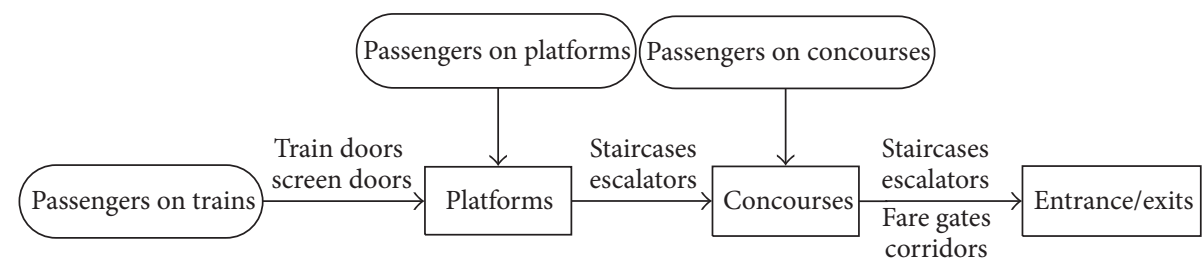

FIGURE 1: Schematic of evacuation direction and route.

safe spots [22]. The basic principle of emergency evacuation is to ensure all passengers are away from being exposed to untenable conditions and fatal accidents so that they are able to reach a safe place through possibly choosing a short and safe evacuation route.

\section{Moving Speed of Evacuees during Evacuation}

There are many psychological and sociological factors, such as age, gender, density of crowd and space facilities, body weight and panic, etc., can affect the moving behaviour of evacuees [16]. The links between moving speed and evacuee density have led to increasing attention and research [23] and focused on the statistical analysis by fitting observed and empirical data.

3.1. Relationship between Speed and Density. The interaction among evacuees due to high density causes the reduction of moving speed [19]. Various types of equations on the relationship between speed and density can be obtained according to field data, such as linear functions, multinomial functions, and exponential functions [19]. In order to ascertain the practical relationship, more than 1550 field data items involving speed and density on the upstairs and corridors are collected through manual accounting from the Xizhimen metro station in Beijing and fitted by linear and exponential functions in the light of different widths of staircases and corridors. The fitting results listed in Table 1 indicate that an exponential function is more suitable to the relationship than a linear function.

3.2. Modification under Emergencies. The movement characteristics of evacuees who have panics in emergency evacuation are different from those in normal situations [19, 24]. The most notable one is that evacuees move or try to move faster than normal. The moving speed, $v_{e}$, in emergency can modified by [24]

$$
v_{e}=\mu_{e} v
$$

where $v$ is the speed of evacuees in normal situations and $\mu_{e}$ represents a coefficient to modify which is calculated by [24]

$$
\mu_{e}= \begin{cases}1.49-0.36 D, & \text { moving in level corridors } \\ 1.26, & \text { going upstairs } \\ 1.21, & \text { going downstairs, }\end{cases}
$$

where $D$ denotes the density of evacuees in terms of projected area (the ratio of evacuee projected to ground area) and is less than $0.92 \mathrm{~m}^{2} / \mathrm{m}^{2}$.

\section{Calculation and Simulation for Emergency Evacuation}

From the perspective of protecting evacuees' life, the calculation and simulation methods on emergency evacuation mainly focus on critical time for safely evacuating as well as the corresponding evacuation capacity from metro stations.. The design codes in some countries prescribe the guaranteed time for evacuation, for example, six minutes in China CDM and NPFA 130 in USA. Some simplifications in the two codes, such as insufficiently considering the relationship between speed and density, propagation of congestion, and interactions among evacuees, lead to the difficulties and inaccuracy for evacuation analysis. To overcome these shortcomings, continuum, network-based, agent-based, and social force models are used to describe evacuation process because they are able to address pedestrian behaviour and crowd dynamics [25]. A control volume model and a state-dependent queue network model are employed to calculate the emergency evacuation in consideration of waiting time and congestion effects due to a large number of evacuees. A multiagentbased simulation approach is developed to introduce the interactions among evacuees and between evacuees and environmental entities in metro stations.

\subsection{Calculation Methods}

4.1.1. China CDM. China CDM was published by the Ministry of Housing and Urban-Rural Development of China through strong practical experiences of designers and engineers, which is applicable to the design, construction, and extension of urban railway systems. There are some regulations and formulas on the evacuation time of passengers. Term 28.2.11 requires that the staircases, escalators, and corridors within public areas in metro stations shall ensure all passengers to safely evacuate from platforms within six minutes in emergency (also called six-minute principle) during peak hours in the far future. The evacuation time, $T_{\mathrm{CDM}}$, is calculated by (3) which is further given in Term 28.2.12 [21].

$$
T_{\mathrm{CDM}}=1+\frac{Q_{1}+Q_{2}}{0.9\left[A_{1}(N-1)+A_{2} B\right]},
$$

where $Q_{1}$ and $Q_{2}$ are the maximum numbers of passengers, respectively, on a train and platforms in the far future; 
TABLE 1: Statistical relationship between upstairs moving speed and density from field data.

\begin{tabular}{|c|c|c|c|c|c|}
\hline \multirow{2}{*}{ Different widths (meter) } & \multirow{2}{*}{ Type of relationship } & \multicolumn{2}{|c|}{ Staircases } & \multicolumn{2}{|c|}{ Corridors } \\
\hline & & Fitting function & $R^{2}$ & Fitting function & $R^{2}$ \\
\hline \multirow{2}{*}{$<2.0$} & Linear & $v=-0.612 \rho+0.702$ & 0.844 & - & - \\
\hline & Exponential & $v=0.711 e^{-0.31 \rho}$ & 0.926 & - & - \\
\hline \multirow{2}{*}{$2.0-3.0$} & Linear & $v=-0.23 \rho+0.91$ & 0.750 & $v=-0.14 \rho+0.71$ & 0.794 \\
\hline & Exponential & $v=0.633 e^{-0.32 \rho}$ & 0.820 & $v=1.306 e^{-0.12 \rho}$ & 0.866 \\
\hline \multirow{2}{*}{$3.0-4.0$} & Linear & $v=-0.295 \rho+1.13$ & 0.864 & $v=-0.09 \rho+0.75$ & 0.870 \\
\hline & Exponential & $v=0.542 e^{-0.17 \rho}$ & 0.905 & $v=1.120 e^{-0.19 \rho}$ & 0.891 \\
\hline \multirow{2}{*}{$4.0-5.0$} & Linear & $v=-0.425 \rho+0.646$ & 0.570 & $v=-0.12 \rho+0.81$ & 0.492 \\
\hline & Exponential & $v=0.652 e^{-0.78 \rho}$ & 0.590 & $v=1.099 e^{-0.52 \rho}$ & 0.504 \\
\hline \multirow{2}{*}{$>5.0$} & Linear & $v=-0.640 \rho+0.791$ & 0.570 & - & - \\
\hline & Exponential & $v=0.801 e^{-0.68 \rho}$ & 0.762 & - & - \\
\hline
\end{tabular}

$A_{1}$ and $A_{2}$ denote the through capacity of evacuees on static escalators and staircases, respectively; $N$ indicates the number of escalators which will be shut down and used as means of evacuation once emergency occurs; $B$ gives the total width of staircases. Equation (3) includes one-minute response time for evacuees consisting of alarming, broadcasting time, and so forth.

\subsubsection{NFPA 130. The NFPA 130, Standard for Fixed Guideway} Transit and Passenger Rail Systems, was approved as an American National Standard [22]. The different editions of NFPA 130 have been widely adopted in many metro systems. NFPA 130 requires that all evacuees safely evacuate not only from platforms but also to safe spots within a given time. Term 5.5.6.1 specifies the threshold capacity of emergency facilities which shall enable all evacuees to evacuate from platforms within four minutes or less. Term 5.5.6.2 designates that stations shall be designed to permit all evacuees to leave from the most remote point on platforms to a safe spot within six minutes or less.

The total evacuation time, $T_{\mathrm{NFPA}}$, is the sum of moving time and waiting time through all sections along the longest evacuation route from platforms and is obtained by

$$
\begin{aligned}
T_{\text {NFPA }}= & T_{\text {walking }}+\max \left(W_{i}^{\text {platform }}\right) \\
& +\max \left(W_{j}^{\text {concourse }}\right),
\end{aligned}
$$

where $T_{\text {walking }}$ is the total walking time of evacuees along the longest evacuation route; $W_{i}^{\text {platform }}$ denotes the waiting time of evacuees at exit $i$ on platforms along it; $W_{j}^{\text {concourse }}$ denotes the waiting time of evacuees at exit $j$ on concourses along it. by

The waiting time at exit $i$ on platforms, $W_{i}^{\text {platform }}$, is given

$$
W_{i}^{\text {platform }}=F_{i}^{\text {platform }}-T_{i}^{\text {platform }},
$$

where $F_{i}^{\text {platform }}$ indicates the passing time of evacuees at exit $i$ on platforms and is obtained by the ratio of the number of evacuees evacuated to the capacity of exit $i . T_{i}^{\text {platform }}$ demonstrates the walking time of evacuees to exit $i$ on platforms. If $T_{i}^{\text {platform }}$ is larger than $F_{i}^{\text {platform }}, W_{i}^{\text {platform }}$ equals zero.
The waiting time at exit $j$ on concourse, $W_{j}^{\text {concourse }}$, is acquired by

$$
W_{j}^{\text {concourse }}=F_{j}^{\text {concourse }}-\max \left(F_{j}^{\text {facility }}\right),
$$

where $F_{j}^{\text {concourse }}$ is the passing time of evacuees at exit $j$ on concourses and $F_{j}^{\text {facility }}$ presents the passing time of evacuees through other facilities to exit $j$ on concourses.

4.1.3. Control Volume Model. The control volume model assumes that each evacuee is an independent particle while neglecting the differences of attributes among individuals. It is able to simulate the queue process of evacuees through a virtual closed surface formed by evacuees when the number of evacuees is larger than the through capacity of facilities. The calculation of evaluation time along an evacuation route is given in (7) through the control volume model after simplification.

$$
\mathrm{ET}=\sum_{i}^{M}\left(\frac{\mathrm{EL}_{i}}{v_{i}}+\frac{\mathrm{CVL}_{i}}{v_{i}}\right)
$$

where $\mathrm{EL}_{i}$ is the length of space or facility $i$ along an evacuation route and $v_{i}$ denotes the velocity of evacuees through space or facility $i$. $\mathrm{CVL}_{i}$ indicates the length of control volume which is formed in space or at facility $i$ and can be obtained by (8). $M$ defines the number of the spaces or facilities along the evacuation route.

$$
\mathrm{CVL}_{i}=\frac{\mathrm{CVA}_{i}}{\mathrm{CVW}_{i}}=\frac{\left(\lambda_{i} \times \mathrm{CVW}_{i}-Q_{i} \times W_{i}\right)}{\left(\mathrm{PA} \times \mathrm{CVW}_{i}\right)},
$$

where $\mathrm{CVA}_{i}$ and $\mathrm{CVW}_{i}$ present the area and width of control volume formed in space or at facility $i$, respectively. $\lambda_{i}$ and $Q_{i}$ demonstrate the evacuee arrival rate at space or facility $i$ and through capacity of space or facility $i$, respectively. $W_{i}$ describes the width of space or facility $i$. PA defines the admissible density of evacuees in space or at facility $i$ which characterizes the maximum degree of congestion during evacuation. 
4.1.4. M/G/C/C State-Dependent Queue Network Model. $\mathrm{M} / \mathrm{G} / \mathrm{c} / \mathrm{c}$ model is able to characterize the service performance of network queuing and the probability of congestion in some queue systems [26]. The notations for the formulation of evacuee queue network are listed as follows.

$N$ : a stochastic variable, denoting the number of evacuees on staircases or in corridors.

$n$ : actual number of evacuees on staircases or in corridors.

$l$ : length of staircases or corridors.

$w$ : width of staircases or corridors.

$k$ : density of evacuees on staircases or in corridors.

$c$ : evacuation capacity on staircases or in corridors which is obtained by $c=\lfloor k l w\rfloor$, where $\lfloor x\rfloor$ represents the largest integer not greater than $x$.

$\lambda$ : arrival rate of evacuees approaching staircases or corridors.

$V_{1}$ : average moving speed of only one evacuee on staircases or in corridors which is fitted by observed data and required to modify by (1) for emergency evacuation.

$V_{n}$ : average moving speed of $n$ evacuees on staircases or in corridors which is fitted by observed data and required to modify by (1) for emergency evacuation.

$f(n)$ : service rate where there is $n$ evacuees on staircases or in corridors.

$p_{n}$ : probability where there is $n$ evacuees on staircases or in corridors; that is, $p_{n}=P_{r}[N=n\}$.

$p_{0}$ : probability where there is not any evacuees on staircases or in corridors; that is, $p_{n}=P_{r}[N=0\}$.

$E\left(T_{1}\right)$ : expected walking time of only one evacuee on staircases or in corridors; that is, $E\left(T_{1}\right)=l / V_{1}$.

The probability, $p_{n}=P_{r}[N=n\}$, where there is $n$ evacuees in queue network systems can be calculated by

$$
\begin{array}{rl}
p_{n}=\left\{\frac{\left[\lambda E\left(T_{1}\right)\right]^{n}}{n ! f(n) f(n-1) \cdots f(2) f(1)}\right\} & p_{0}, \\
n & n=0,1,2, \ldots, c,
\end{array}
$$

where $p_{0}$ is given through

$$
p_{0}{ }^{-1}=1+\sum_{i=1}^{c}\left\{\frac{\left[\lambda E\left(T_{1}\right)\right]^{i}}{i ! f(i) f(i-1) \cdots f(2) f(1)}\right\} .
$$

Two important criteria employed to evaluate the evacuation bottleneck of evacuees on staircases or in corridors are proposed as follows:

$$
\begin{aligned}
p_{c} & =p_{r}[N=c] \\
\theta & =\lambda\left(1-p_{c}\right),
\end{aligned}
$$

where $p_{c}$ is the probability of passenger congestion occurring on staircases or in corridors, $\theta$ defines the output evacuee number per unit time from the queue network.
4.2. Multiagent-Based Simulation. Simulation is widely employed to evaluate evacuation problems and usually classified into macroscopic and microscopic simulation. Macroscopic simulation mainly focuses on an overall situation and assumes aggregated values while microscopic aspect pays attention to the behaviour of individuals, that is, the movements of individuals and their interactions [27]. The interactions among evacuees as well as between evacuees and environment involve not only space, time, and information exchange but also human decision and behaviours. The multiagent-based technique is a capable approach to handle them [28]. The origin of multiagent system in computer science was found during the early 1990s. It quickly becomes the key issue in various areas which many researchers pay much attention to. The basic elements of a multiagent system contain environment, sensors, knowledge base, decision rules, and actuators.

4.2.1. Construction of Multiagent Simulation Model. Evacuees and their behaviours during evacuation from stations in multiagent model are realized through many agents who are usually able to perceive environments or other entities, make decisions according to given rules, and take actions to realize specific goals or tasks. Evacuee agents can complete their actions during a simulation process according to social force model [29] in which the self-driving force to move has to be involved for each evacuee agent and the repulsive, extrusion, and friction forces will be considered if evacuee agents are sufficiently close or touch environmental entities or other agents. The social force can be calculated according to the formulation from [29].

A general multiagent simulation flowchart is developed in Figure 2 to model the emergency evacuation of evacuees from metro stations. The movement velocity of evacuees is modified by (1) and (2) in Section 3.2 when emergency evacuation occurs.

4.2.2. Implementation. There are many tools to implement the simulation supported by multiagent technique in addition to commercially available models, like StarLogo, Swarm Ascape, and AnyLogic. AnyLogic is a powerful tool to simulate the emergency evacuation of evacuees from metro stations as it is able to provide complex solutions through a multiagent-based and discrete event special pedestrian movement simulation module to plan, manage, and evaluate evacuee flow in public buildings like airports and railway stations. The pedestrian library is selected to construct the simulation environment including platforms, entities (walls, pillars, stairs, and escalators) and events (simulation clock and evacuation generators) as well to reproduce the movement process of evacuees in metro stations. A simulation model for the emergency evacuation from a metro station is developed by AnyLogic in Figure 3.

\section{Case Studies}

The Xizhimen metro station covering an area of 4.24 hectares, one of the large transfer metro stations in Beijing, is selected in case studies for verifying the feasibility of the 


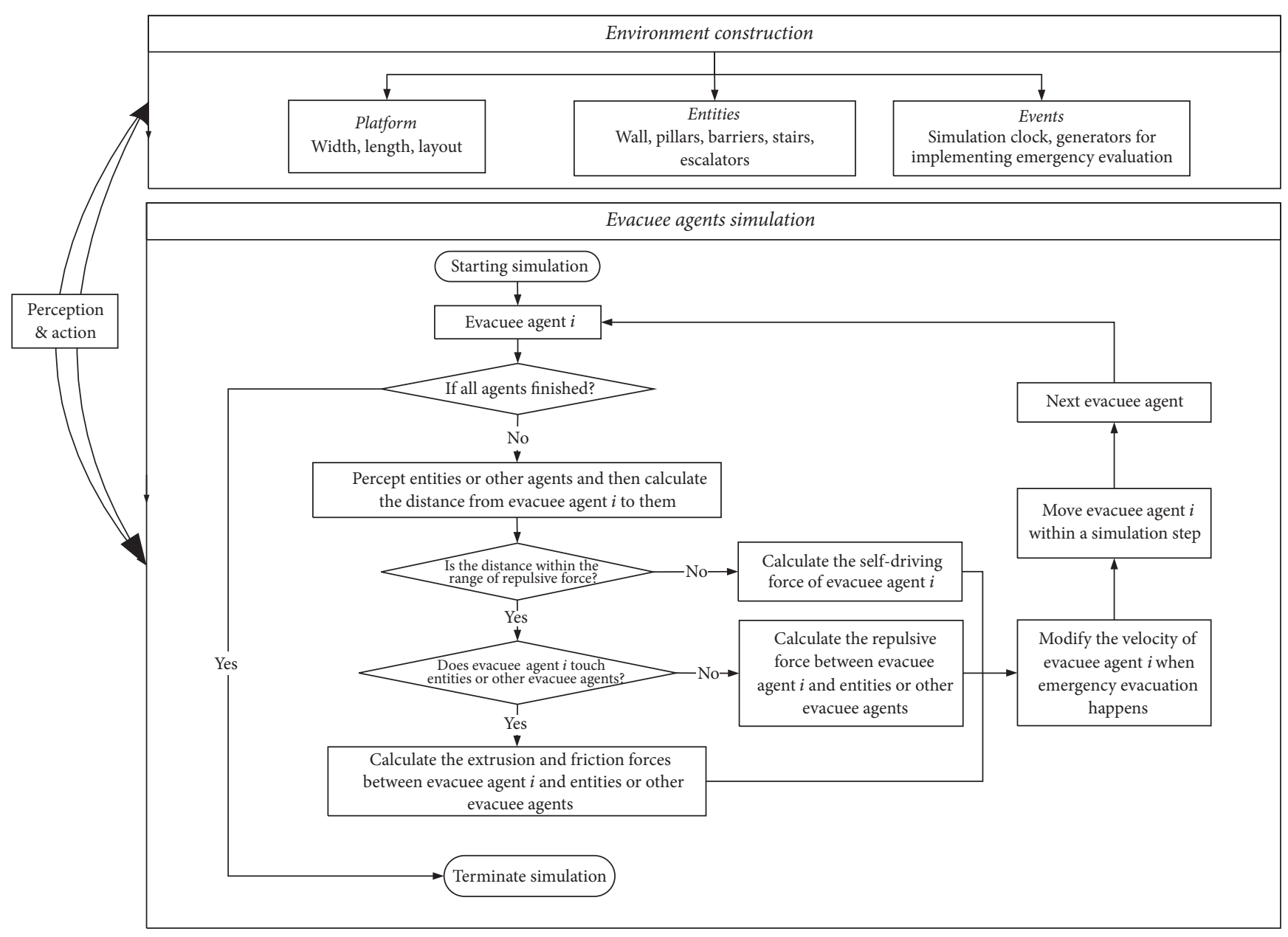

FIGURE 2: Simulation flowchart of evacuation from metro stations through multiagent technique.

methodologies in this study. Metro Line 2, Line 4, and Line 13 interchange at the Xizhimen metro station which is adjacent to the Beijing north railway station. More than 400 thousands of passengers per day are served here [30]. The layout of station and physical characteristics of facilities including corridors, staircases, and escalators are investigated through a field interview method.

\subsection{Set-Up}

5.1.1. Layout of Station. The range of research here is confined within the underground platforms, concourses, and corridors of Line 2 and Line 4 because the station of Line 13 is far separated and connected by long corridors. The layout of the part to be studied is described as shown in Figure 4 and the evacuation facilities include 20 staircases (S1 to S20), 18 corridors ( $\mathrm{C} 1$ to $\mathrm{C} 18), 9$ escalators (E1 to E9), 5 exits (A1, A2, $\mathrm{B}, \mathrm{C}$, and $\mathrm{D})$, and 6 groups of fare gates (G1-G6).

5.1.2. Physical Characteristics of Facilities. Corridors, staircases, and escalators are the most crucial facilities for evacuees in metro stations. The physical characteristics of facilities can affect the evacuation choice and evacuation performance of evacuees. The exponential relationship between the speed and density of evacuees in corridors and on staircases are fitted due to the collected data. The two speed ranges modified by (1) in emergency locate within the two intervals $[1.10 \mathrm{~m} / \mathrm{s}, 2.05 \mathrm{~m} / \mathrm{s}]$ and $[0.66 \mathrm{~m} / \mathrm{s}, 1.23 \mathrm{~m} / \mathrm{s}]$, respectively, for corridors and the upstairs.

5.1.3. Evacuation Scenarios. Some evacuation scenarios in emergency are considered here according to the different accidents and evacuation strategies. These scenarios are commonly divided into the accidents on concourses and platforms in light of the differences of starting positions and safe spots. The evacuation results of two scenarios obtained by the China CDM, NFPA 130, control volume model, M/G/c/c model, and multiagent-based model are further compared and analysed. The scenarios for the emergency evacuation from a platform (Scenario 1) and concourse (Scenario 2) are set up here. The evacuation routes represented in Figures 5 and 6 are strictly defined as starting from the platform and ending at the exits which are indeed safe spots for underground stations. The assumptions include that the evacuees are uniformly distributed on platforms and choose their evacuation routes to safe spots with an equal probability. 

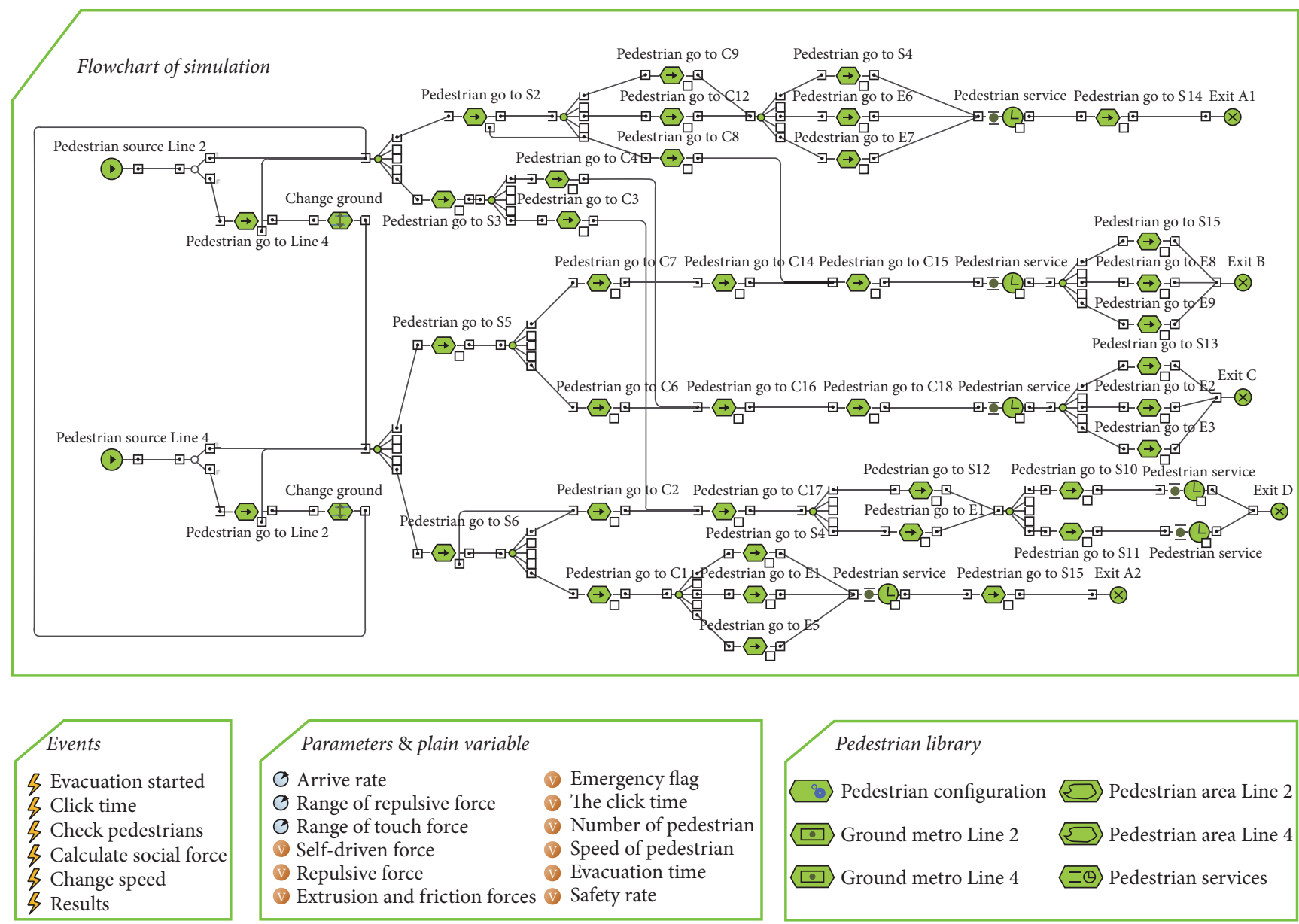

FIgURE 3: Implementation of evacuation in case studies by multiagent simulation.

The impacts on evacuation from the features of accidents, like smoke, are not considered here as they require another intensive study.

Two widely used criteria to assess evacuation are imported here, the evacuation time with a given number of evacuees and the number of evacuees within a given time. The evacuees include the passengers in a full loaded train and waiting and walking in the station according to China CDM. Due to the most adverse condition where two trains arrive at the same time, there will be 3360 passengers in two trains with six units and 200 passengers in corridors or on staircases to be evacuated. The multiagent-based simulation are first used to obtain the evacuation time of each route and total evacuation time when the arrival rates of evacuees on platforms which can be obtained through a filed counting method vary from 6 to 14 persons per second.

5.2. Simulation Results. The evacuation time in two scenarios is attained through the simulation model as displayed, respectively, in Figures 7 and 8. The evacuation times through different routes at various arrival rates from Figures 7 and 8 vary from 130 to 270 seconds, which are obviously larger than those values within 80 and 170 seconds acquired by the former methodologies. The routes with longest evacuation time are Routes 7 and 3, respectively, in Scenarios 1 and 2 and also totally different from the other four methodologies. The results mainly show that the multiagent-based simulation approach additionally takes into account the existing interactions among evacuees and the influences between evacuees and environmental entities and that as a consequence the evacuation time prediction is extended.

\subsection{Comparisons and Analysis}

5.3.1. Evacuation Time. China CDM and NFPA 130 are both able to judge whether the total evacuation time for the given number of evacuees satisfies "six-minute principle" or not. NFPA 130 also gives the total evacuation time of the longest evacuation route rather than each evacuation route in metro stations under the most adverse conditions or maximum time requirements. However, both of them do not consider the influences of different arrival intensities and waiting time of evacuees due to the insufficient capacity of facilities. The control volume and $\mathrm{M} / \mathrm{G} / \mathrm{c} / \mathrm{c}$ models provide the evacuation time of each route with consideration of the aforementioned waiting time of evacuees. Moreover, the M/G/c/c model also involves the effects on movement speed by evacuee density. The interactions relating to evacuees further considered by 


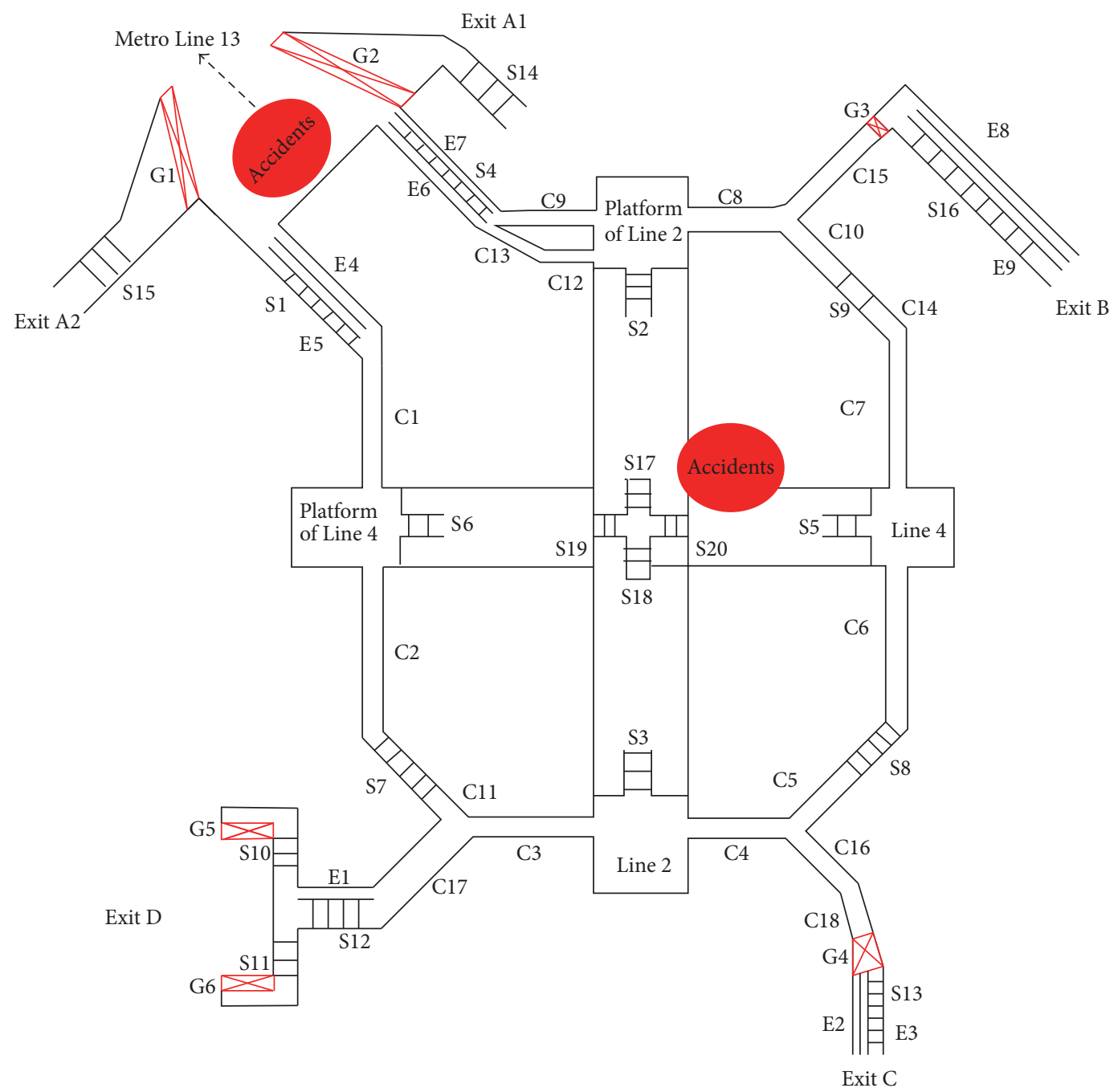

FIGURE 4: Layout of the part to be studied in the Xizhimen station.

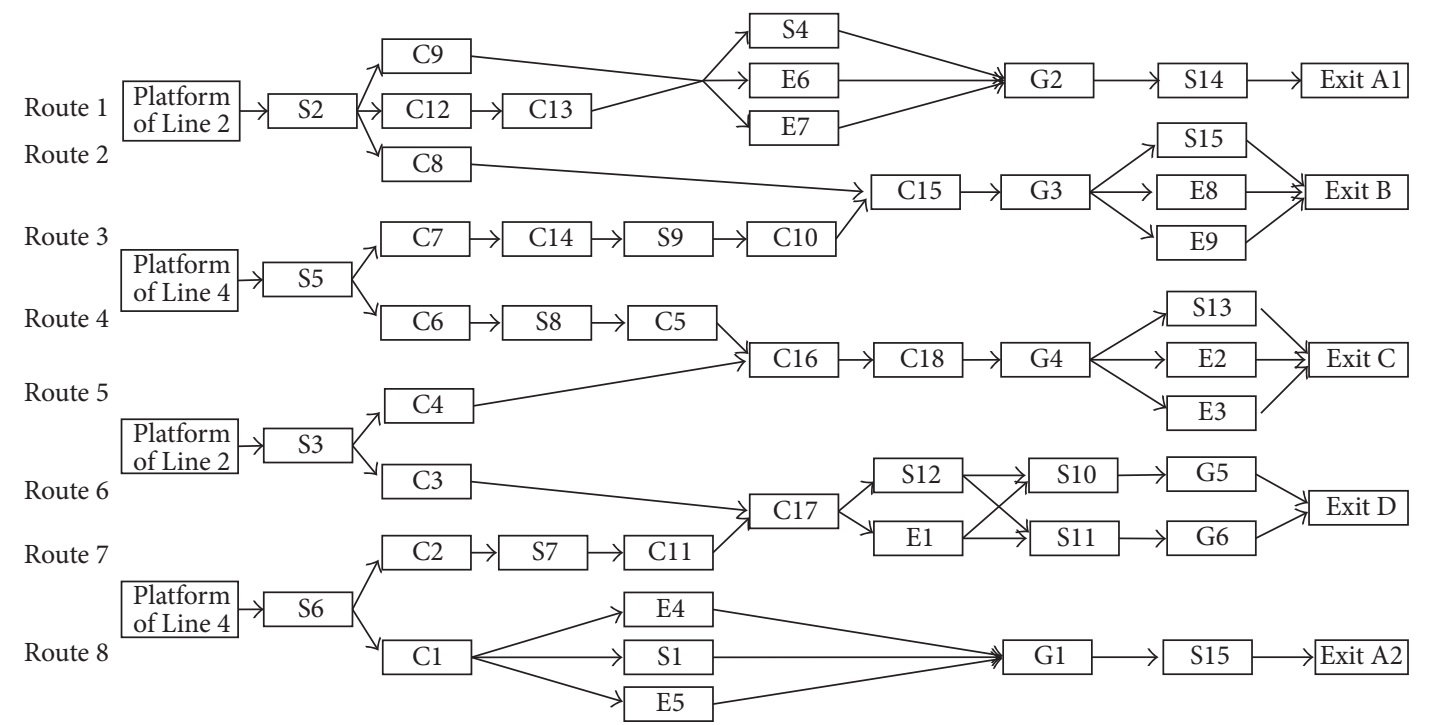

FIGURE 5: Evaluation routes when accidents occur in platform (Scenario 1). 


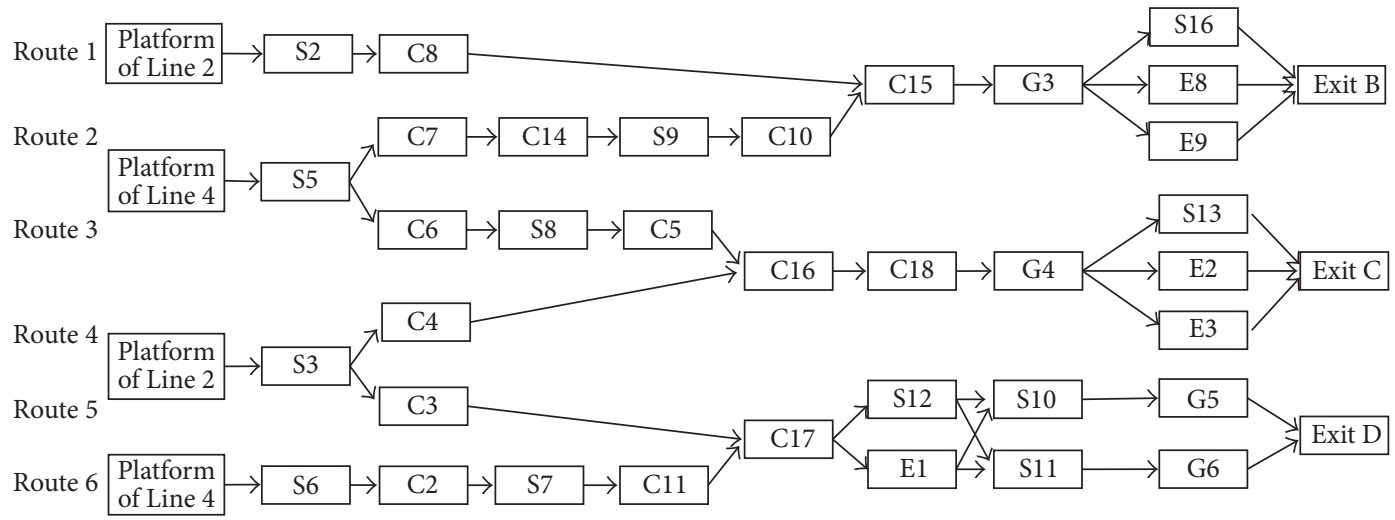

FIGURE 6: Evaluation routes when accidents occur in concourse (Scenario 2).

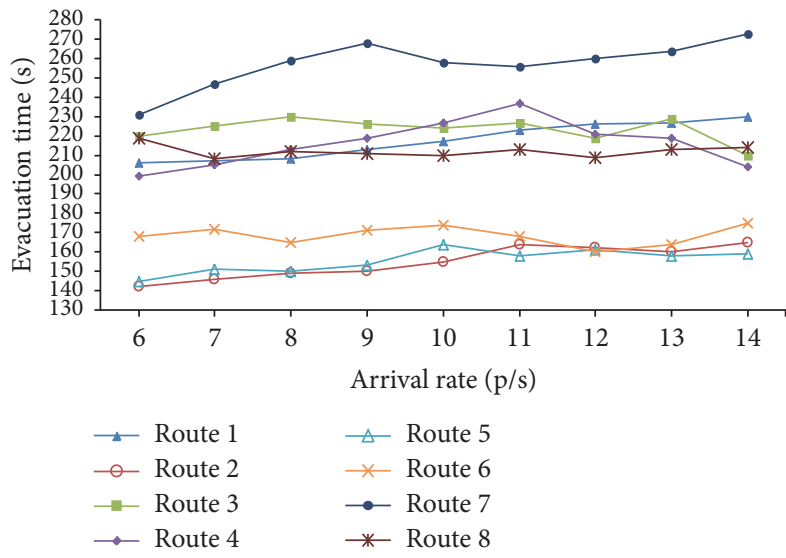

FIGURE 7: Evacuation time of different routes through multiagent simulation in Scenario 1.

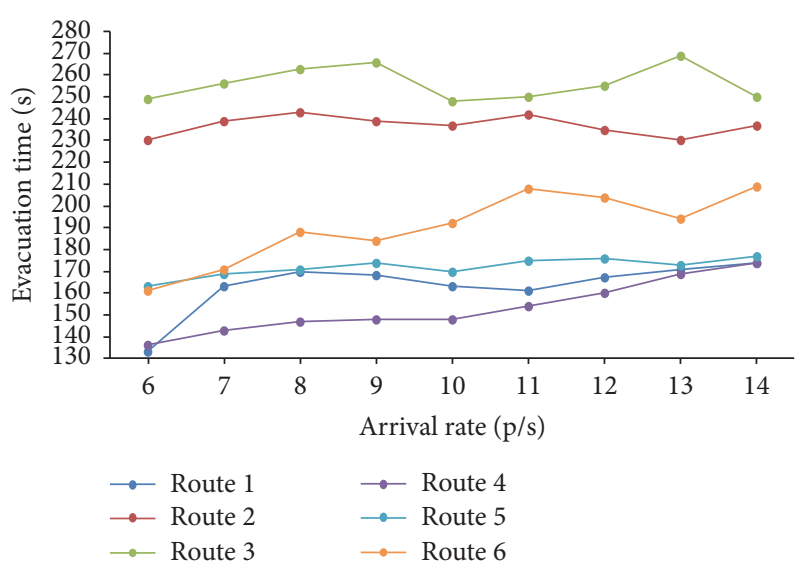

FIGURE 8: Evacuation time of different routes through multiagent simulation in Scenario 2.

the multiagent-based simulation actually lead to the retardarce of evacuees movement and require more evacuation time.

5.3.2. Evacuee Number. The number of evacuees in Scenarios 1 and 2 from five methodologies under the different arrival

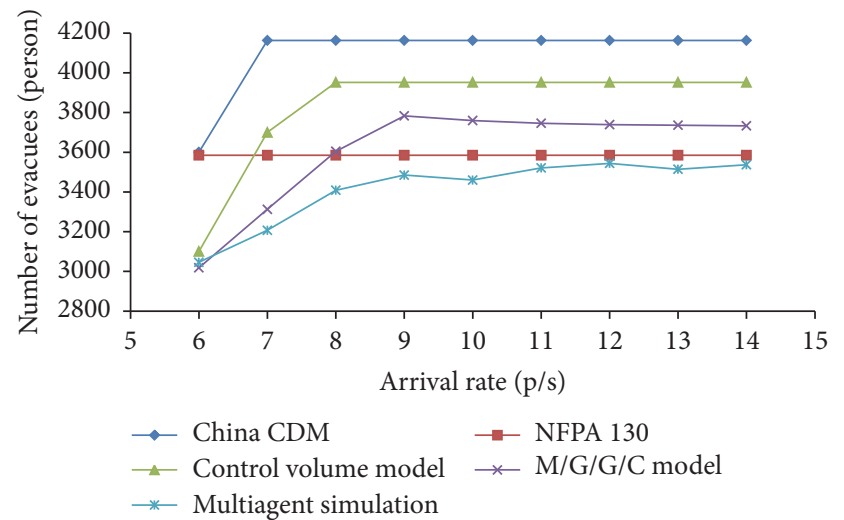

FIGURE 9: Number of evacuees within 6 minutes through five methodologies in Scenario 1.

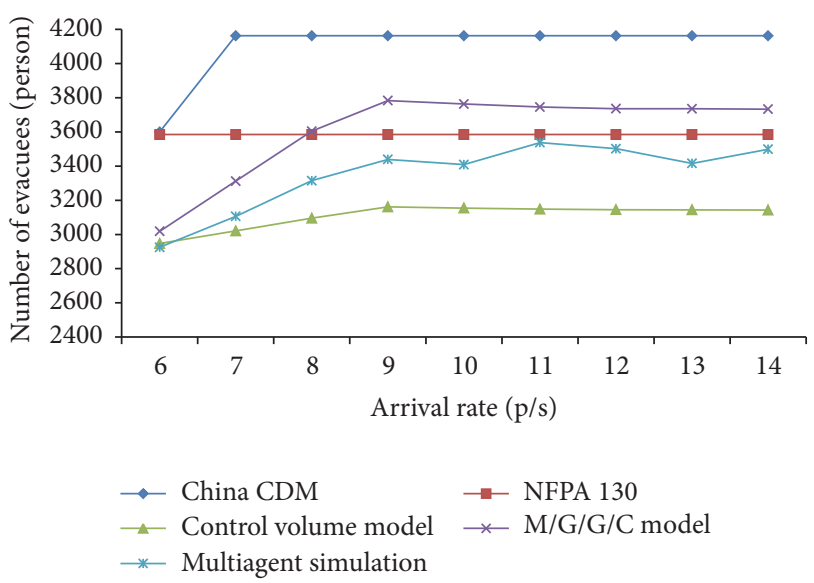

Figure 10: Number of evacuees within 6 minutes through five methodologies in Scenario 2.

rates of evacuees are represented, respectively, in Figures 9 and 10 .

The same maximum evacuee numbers of 4163 persons by China CDM are obtained in two scenarios only by calculating the number of evacuees through the staircases connecting the platforms rather than arriving at exits when the arrival rate is 
larger than $7 \mathrm{p} / \mathrm{s}$, which produces more evacuees than other methodologies do. NFPA 130 gives the same critical evacuee numbers of 3583 persons within six minutes considering the most adverse conditions (the longest evacuation routes) in two scenarios. The number of evacuees from the control volume model increases until the arrival rate is larger than $9 \mathrm{p} / \mathrm{s}$ and reaches the critical numbers of 3952 and 3161 persons, respectively, in Scenarios 1 and 2. The $M / G / c / c$ model provides the same number trends of evacuees in two scenarios that first increase with the arrival rate reaching the critical number of 3783 persons when the arrival rate is $9 \mathrm{p} / \mathrm{s}$ and then slightly decrease as the high density of evacuee causes the congestion and the downgrade of evacuation performance. The number of evacuees from the multiagentbased simulation in two scenarios is fluctuating around 3500 persons and reaches the maximum evacuation numbers of 3544 and 3538 persons, respectively, in Scenarios 1 and 2 when the arrival rate is larger than $9 \mathrm{p} / \mathrm{s}$.

The number of evacuees from the control volume model in two scenarios varies much because of the change of accident positions as the method concerns all spaces or facilities along each evacuation route while the other four methodologies pay more attention to the critical capacity of spaces or facilities and influence factors. For example, China CDM assumes that the staircases connecting platforms are the most crucial component to decide the evacuee number while NFPA 130 focuses more on the longest one among all evacuation routes. The variances of evacuation routes in two scenarios do not actually affect the critical spaces, facilities, or influence factors like the density and interactions of evacuees.

5.3.3. Evacuation Bottlenecks. The congestion probability of evacuation can be calculated by (11) in the M/G/c/c model and the multiagent-based simulation, which identifies the evacuation bottlenecks of evacuees for the scenarios in the cases studies if it is more than 0.5. The congestion probabilities of evacuation occurring on S2, S3, S5, and S6 in Scenarios 1 and 2 increase with the arrival rates of evacuees. The $\mathrm{M} / \mathrm{G} / \mathrm{c} / \mathrm{c}$ reveals that the congestion of evacuees occurs successively on four staircases, S3, S6, S2, and S5, without consideration of the complex interactions of evacuees. The results from the multiagent-based simulation demonstrate the almost consistent occurrence of congestion due to the dynamic interactions and influence among evacuees when the arrival rate of evacuees is larger than $9 \mathrm{p} / \mathrm{s}$. The two results both indicate that the staircases connecting platforms are the evacuation bottlenecks in the Xizhimen metro station.

\section{Conclusions}

The calculation and simulation on emergency evacuation is fundamental to ensure reasonable design, efficient operation, and low risk management for metro systems. Five methodologies are introduced to attain the evacuation performances in this study. Some conclusions are summarized as below from the comparisons and analysis on the results from case studies.

The involvement of different methodologies is dependent on how users treat the impact factors and strategies of evacuation. China CDM and NFPA 130 propose two concise methodologies which only consider the most critical facilities and the most adverse evacuation conditions and usually provide high and low values of evacuee number within given evacuation time, respectively. The other methodologies regard the exits of metro stations as evacuation terminus or safe spots while the evacuees will be safe in China $\mathrm{CDM}$ if they leave platforms. The control volume, $\mathrm{M} / \mathrm{G} / \mathrm{c} / \mathrm{c}$, and multiagent-based models introduce the geometry and capacity of facilities, relationship between density and speed, and interactions among evacuees and are able to reflect the waiting time, congestion extent, and evacuation bottlenecks through analysing the efficiency of each evacuation route.

Five methodologies have their individual superiorities in design, management, and technical application. China CDM and NFPA 130, as the national design standards, are two authoritative criteria and efficient quantitative methods for planners and designers to obtain evacuation requirements. The control volume, $\mathrm{M} / \mathrm{G} / \mathrm{c} / \mathrm{c}$, and multiagent-based models are helpful to assess whole evacuation process, especially when the multiagent-based approach is used to incorporate more details during evacuation for operation improvement and risk management. Modelling details on the psychological characteristics of evacuees are required in the further research work to describe the nervous emotion or panic during emergency evacuation.

\section{Conflicts of Interest}

The authors declare that they have no conflicts of interest.

\section{Acknowledgments}

The authors are grateful to the Beijing Natural Science Foundation (Grant no. 9152013), the Fundamental Research Funds for the Central Universities of China (Grant no. 2016JBZ001), and National Natural Science Foundation of China (Grant no. 71571015) for their financial support.

\section{References}

[1] W. H. Hong, "The progress and controlling situation of Daegu Subway fire disaster," in Proceedings of the 6th Asia-Oceania Symposium on Fire Science and Technology, pp. 17-20, 2004.

[2] D. Helbing, I. Farkas, and T. Vicsek, "Simulating dynamical features of escape panic," Nature, vol. 407, no. 6803, pp. 487490, 2000.

[3] J. Shi, A. Ren, and C. Chen, "Agent-based evacuation model of large public buildings under fire conditions," Automation in Construction, vol. 18, no. 3, pp. 338-347, 2009.

[4] H.-Y. Lee, I.-T. Yang, and Y.-C. Lin, "Laying out the occupant flows in public buildings for operating efficiency," Building and Environment, vol. 51, pp. 231-242, 2012.

[5] S. Gwynne, E. R. Galea, M. Owen, P. J. Lawrence, and L. Filippidis, "A review of the methodologies used in the computer simulation of evacuation from the built environment," Building and Environment, vol. 34, no. 6, pp. 741-749, 1999.

[6] Y. Zheng, B. Jia, X. G. Li, and N. Zhu, "Evacuation dynamics with fire spreading based on cellular automaton," Physica A: 
Statistical Mechanics and Its Applications, vol. 390, no. 18-19, pp. 3147-3156, 2011.

[7] S. Kalakou and F. Moura, "Bridging the gap in planning indoor pedestrian facilities," Transport Reviews, vol. 34, no. 4, pp. 474500,2014

[8] Z. Zainuddin and M. Shuaib, "Modification of the decisionmaking capability in the social force model for the evacuation process," Transport Theory and Statistical Physics, vol. 39, no. 1, pp. 47-70, 2010.

[9] T. Saelao and S. Patvichaichod, "The computational fluid dynamic simulation of fire evacuation from the student dormitory," American Journal of Applied Sciences, vol. 9, no. 3, pp. 429435, 2012

[10] S. M. Lo, H. C. Huang, P. Wang, and K. K. Yuen, "A game theory based exit selection model for evacuation," Fire Safety Journal, vol. 41, no. 5, pp. 364-369, 2006.

[11] C. Saloma, G. J. Perez, G. Tapang, M. Lim, and C. PalmesSaloma, "Self-organized queuing and scale-free behavior in real escape panic," Proceedings of the National Academy of Sciences of the United States of America, vol. 100, no. 21, pp. 11947-11952, 2003.

[12] M. Tsukahara, Y. Koshiba, and H. Ohtani, "Effectiveness of downward evacuation in a large-scale subway fire using Fire Dynamics Simulator," Tunnelling and Underground Space Technology, vol. 26, no. 4, pp. 573-581, 2011.

[13] A. Haack and J. Schreyer, "Emergency scenarios for tunnels and underground stations in public transport," Tunnelling and Underground Space Technology, vol. 21, no. 3-4, pp. 285-294, 2006.

[14] C. S. Jiang, Y. F. Deng, C. Hu, H. Ding, and W. K. Chow, "Crowding in platform staircases of a subway station in China during rush hours," Safety Science, vol. 47, no. 7, pp. 931-938, 2009.

[15] C. S. Jiang, F. Yuan, and W. K. Chow, "Effect of varying two key parameters in simulating evacuation for subway stations in China," Safety Science, vol. 48, no. 4, pp. 445-451, 2010.

[16] M. Zhong, C. Shi, X. Tu, T. Fu, and L. He, "Study of the human evacuation simulation of metro fire safety analysis in China," Journal of Loss Prevention in the Process Industries, vol. 21, no. 3, pp. 287-298, 2008.

[17] C. Shi, M. Zhong, X. Nong, L. He, J. Shi, and G. Feng, "Modeling and safety strategy of passenger evacuation in a metro station in China," Safety Science, vol. 50, no. 5, pp. 1319-1332, 2012.

[18] R. Alizadeh, "A dynamic cellular automaton model for evacuation process with obstacles," Safety Science, vol. 49, no. 2, pp. 315-323, 2011.

[19] V. V. Kholshevnikov, T. J. Shields, K. E. Boyce, and D. A. Samoshin, "Recent developments in pedestrian flow theory and research in Russia," Fire Safety Journal, vol. 43, no. 2, pp. 108-118, 2008.

[20] F. Zheng, J. P. Yuan, Y. C. Wang, and S. M. Lo, "Survey of pedestrian movement and development of a crowd dynamics model," Fire Safety Journal, vol. 43, no. 6, pp. 459-465, 2008.

[21] Ministry of Housing and Urban-Rural Development of the People's Republic of China, "Code for design of metro," GB 501572013, Ministry of Housing and Urban-Rural Development of the People's Republic of China, Beijing, China, 2013, 2013 (Chinese).

[22] National Fire Protection Association, "Standard for fixed guideway Transit and passenger rail systems," Tech. Rep. 130, 2010.

[23] A. Gupta and N. Pundir, "Pedestrian flow characteristics studies: a review," Transport Reviews, vol. 35, no. 4, pp. 445-465, 2015.
[24] V. M. Predtechenskii and A. I. Milinskii, Planning for Foot Traffic Flow in Buildings, Amerind Publishing, New Delhi, India, 1978.

[25] H. Vermuyten, J. Beliën, L. De Boeck, G. Reniers, and T. Wauters, "A review of optimisation models for pedestrian evacuation and design problems," Safety Science, vol. 87, pp. 167178, 2016.

[26] J. MacGregor Smith and W.-J. Li, "Quadratic assignment problems and $\mathrm{M} / \mathrm{G} / \mathrm{C} / \mathrm{C} /$ state dependent network flows," Journal of Combinatorial Optimization, vol. 5, no. 4, pp. 421-443, 2001.

[27] A. Borrmann, A. Kneidl, G. Köster, S. Ruzika, and M. Thiemann, "Bidirectional coupling of macroscopic and microscopic pedestrian evacuation models," Safety Science, vol. 50, no. 8, pp. 1695-1703, 2012.

[28] Y. M. Chen and B.-Y. Wang, "A study on modeling of human spatial behavior using multi-agent technique," Expert Systems with Applications, vol. 39, no. 3, pp. 3048-3060, 2012.

[29] D. Helbing and P. Molnár, "Social force model for pedestrian dynamics," Physical Review E, vol. 51, no. 5, pp. 4282-4286, 1995.

[30] China Association of Metros, 2016 Statistical Report for Urban Rail Transit in China, China Association of Metros, Beijing, China, 2017, 2017 (Chinese). 


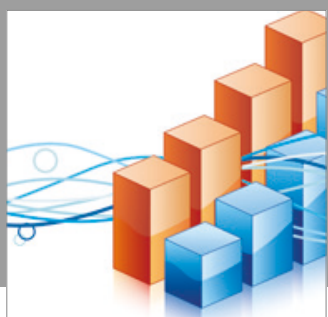

Advances in

Operations Research

vatersals

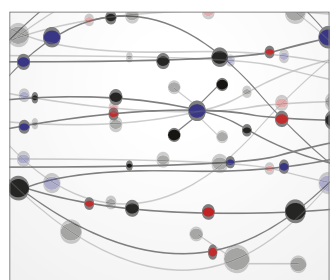

\section{The Scientific} World Journal
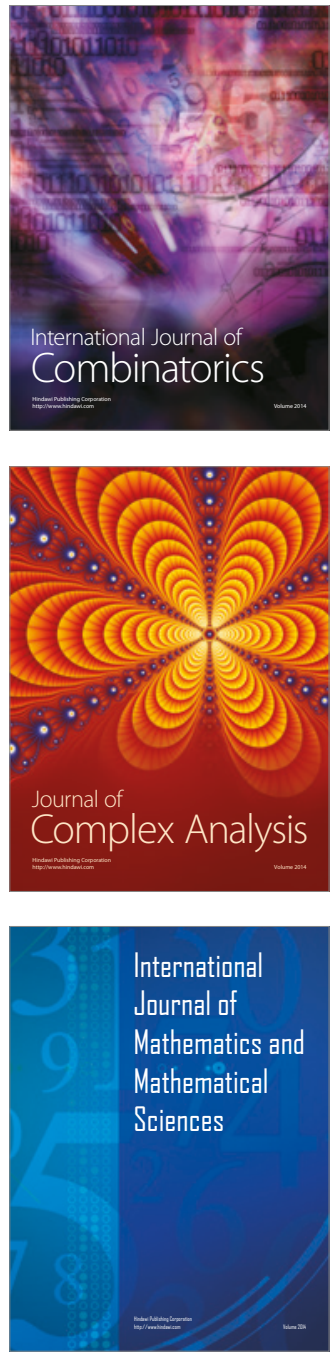
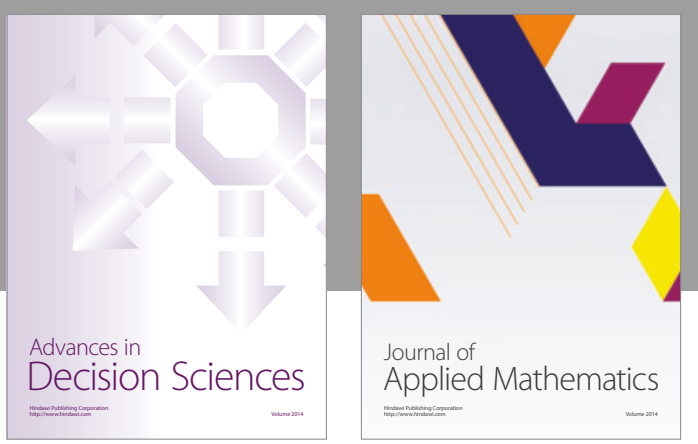

Algebra

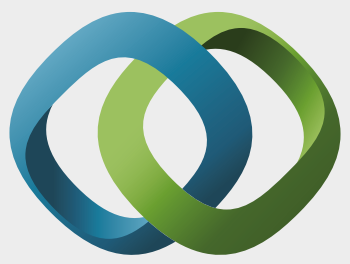

\section{Hindawi}

Submit your manuscripts at

https://www.hindawi.com
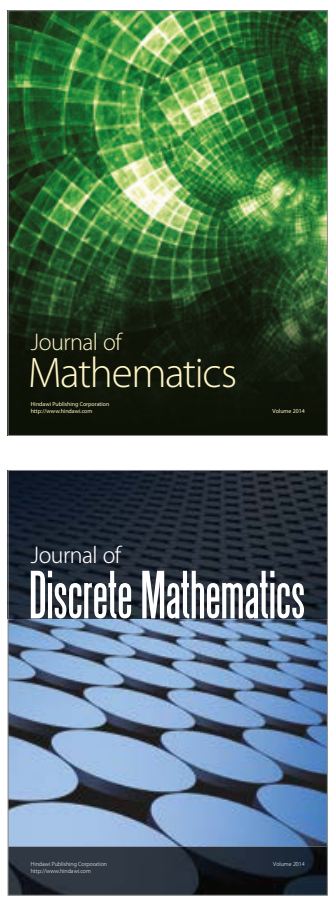

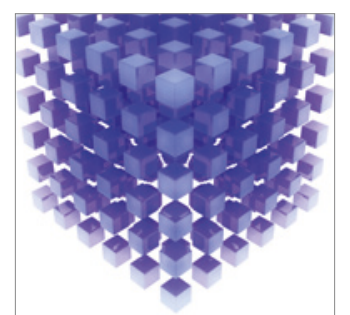

Mathematical Problems in Engineering
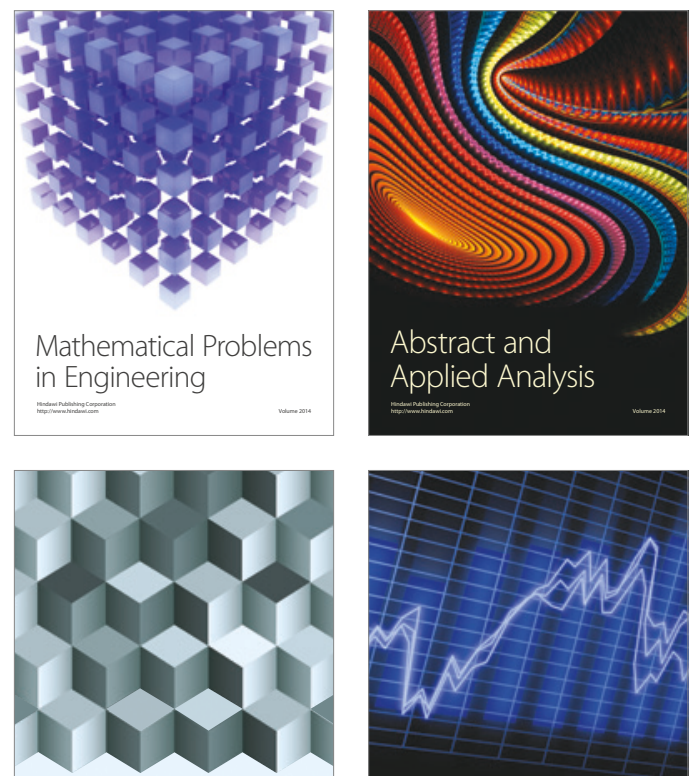

Journal of

Function Spaces

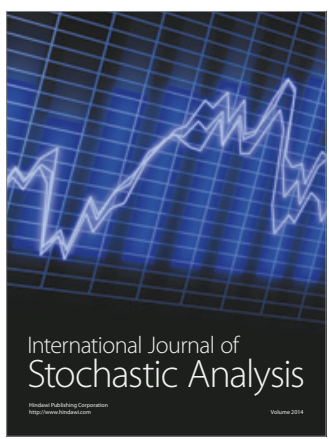

Probability and Statistics
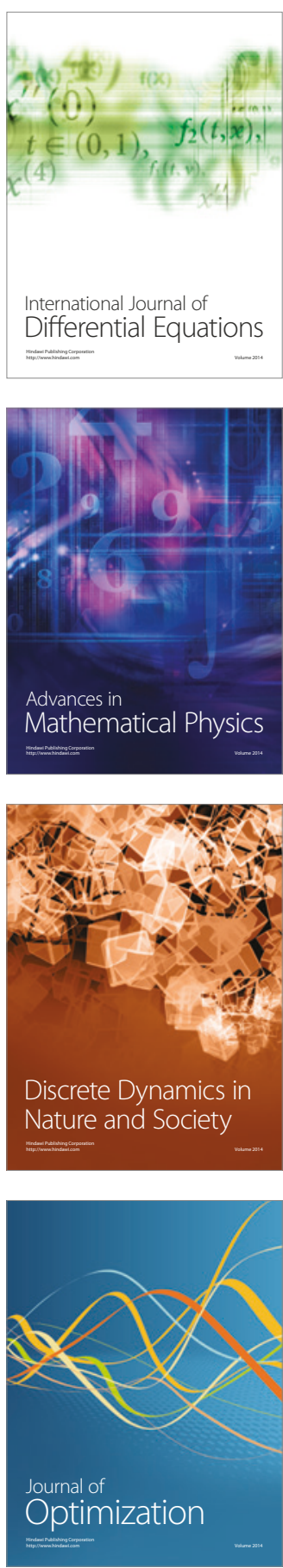